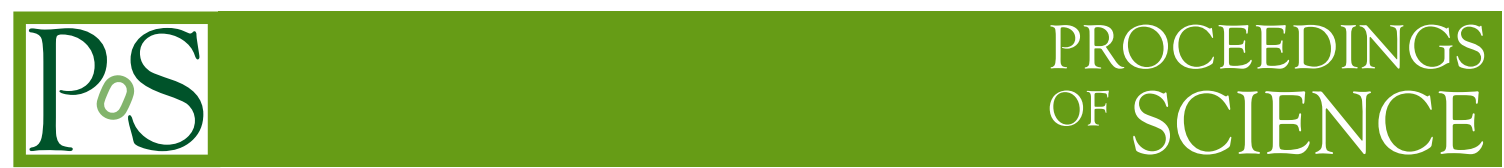

\title{
Study of charmonium decays at BESIII
}

\author{
Elisa Fioravanti* \\ INFN Ferrara, Via Saragat 1, 44122 Ferrara, Italy \\ E-mail: fioravanti@fe.infn.it
}

We present new results on charmonium decays from the BESIII experiment located at the Beijing

Electron Positron Collider II.

VIII International Workshop On Charm Physics

5-9 September, 2016

Bologna, Italy

${ }^{*}$ Speaker. 


\section{Measurement of the leptonic decay width of $J / \psi$ using Initial State Radiation}

The electronic width of the $J / \psi$ resonance $\Gamma_{e e}=\Gamma_{e e}(J / \psi)$ has been measured by BABAR [1] and CLEO-c [2] using the Initial State Radiation (ISR) method. Also the KEDR experiment, measured its electronic width with improved precision [3] using a different method.

We study the process $e^{+} e^{-} \rightarrow J / \psi \gamma \rightarrow \mu^{+} \mu^{-} \gamma$ using the Initial State Radiation method analyzing $2.93 \mathrm{fb}^{-1}$ of data taken at $\sqrt{s}=3.773 \mathrm{GeV}$ [4] with the BESIII detector at the BEPCII collider.

The cross section $\sigma_{J / \psi \gamma}=\sigma\left(e^{+} e^{-} \rightarrow J / \psi \gamma \rightarrow \mu^{+} \mu^{-} \gamma\right)$ is proportional to $\Gamma_{e e} \cdot \mathscr{B}_{\mu \mu}$ where $\mathscr{B}_{\mu \mu}=$ $\mathscr{B}\left(J / \psi \rightarrow \mu^{+} \mu^{-}\right)$is the branching fraction of the muonic decay of the $J / \psi$ resonance. With the precise measurement of $\mathscr{B}_{\mu \mu}$ from BESIII [5] we have the opportunity to obtain $\Gamma_{e e}$ with high precision.

We make use of an iteration method, using the PDG 2014 value, as starting one, to extract the measurement of $\Gamma_{e e} \cdot \mathscr{B}_{\mu \mu}$. The obtained output value is $\Gamma_{e e} \cdot \mathscr{B}_{\mu \mu}=333.4 \pm 2.5 \mathrm{eV}$. The fit to the invariant mass of $\mu^{+} \mu^{-}$using this value is shown in Figure 1.

With $\mathscr{B}_{\mu^{+} \mu^{-}}=(5.973 \pm 0.007 \pm 0.038) \%$ from an independent BESIII measurement [5], our measurement yields $\Gamma_{e e}=(5.58 \pm 0.05 \pm 0.08) \mathrm{KeV}$. Our measurement of $\Gamma_{e e} \cdot \mathscr{B} \mu \mu$ is consistent with the results from BABAR [1], CLEO-c [2] and KEDR [3]. The measured value for $\Gamma_{e e}$ is more precise, as summarized in Table 1.

Table 1: Results of the BABAR, CLEO-c and KEDR measurements compared to this work.

\begin{tabular}{lrrr}
\hline Measurement & $\Gamma_{e e} \cdot \mathscr{B}_{\mu \mu}[\mathrm{eV}]$ & Used $\mathscr{B}_{\mu \mu}$ value [\%] & $\Gamma_{e e}[\mathrm{keV}]$ \\
\hline BABAR & $330.1 \pm 7.7 \pm 7.3$ & $5.88 \pm 0.10$ & $5.61 \pm 0.20$ \\
CLEO-c & $338.4 \pm 5.8 \pm 7.1$ & $5.953 \pm 0.056 \pm 0.042$ & $5.68 \pm 0.11 \pm 0.13$ \\
KEDR & $331.8 \pm 5.2 \pm 6.3$ & $5.94 \pm 0.06$ & $5.59 \pm 0.12$ \\
This work & $333.4 \pm 2.5 \pm 4.4$ & $5.973 \pm 0.007 \pm 0.037$ & $5.58 \pm 0.05 \pm 0.08$ \\
\hline
\end{tabular}

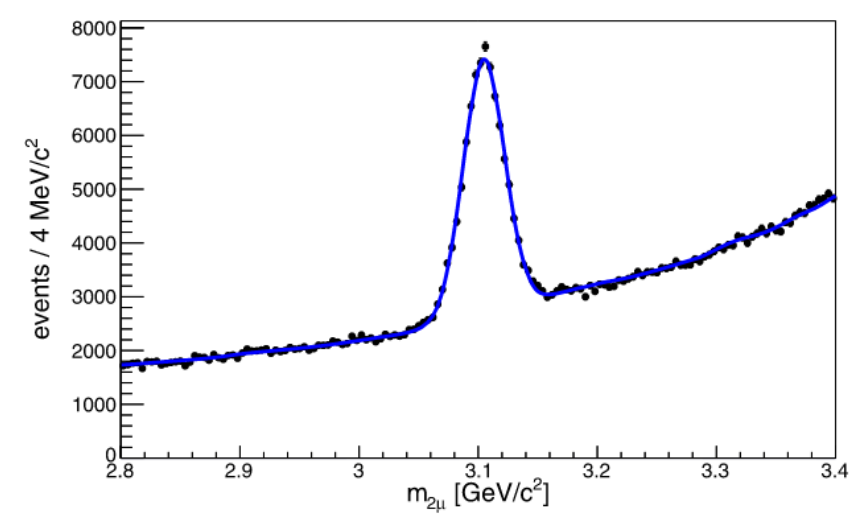

Figure 1: Fit to the invariant mass distribution $\mu^{+} \mu^{-}$on the data.

\section{Observation of $h_{c}$ radiative decay $h_{c} \rightarrow \gamma \eta^{\prime}$ and evidence for $h_{c} \rightarrow \gamma \eta$}

Charmonium, the bound state of a charmed quark and anticharmed quark $(c \bar{c})$, has played an 
important role for our understanding of quantum chromodynamics (QCD), which is fundamental theory that describes the strong interactions between quarks and gluons. All charmonium states below the open-charm $D \bar{D}$ threshold have been observed experimentally and can be well described by potential models. However knowledge is still sparse on the $P$-wave spin-singlet state, $h_{c}\left({ }^{1} P_{1}\right)$. So far, only a few decay modes of $h_{c}$ have been observed, in particular, the radiative transition $h_{c} \rightarrow \gamma \eta_{c}(\mathscr{B} \sim 50 \%)$ and one hadronic decay $h_{c} \rightarrow 2\left(\pi^{+} \pi^{-}\right) \pi^{0}(\mathscr{B} \sim 2 \%)$. Searches for the new $h_{c}$ decay modes, such as $h_{c} \rightarrow \gamma \eta\left(\eta^{\prime}\right)$, are useful for providing constraints to theoretical models in the charmonium region. The ratio of the branching fraction $\mathscr{B}\left(h_{c} \rightarrow \gamma \eta\right)$ over $\mathscr{B}\left(h_{c} \rightarrow \gamma \eta^{\prime}\right)$ can also be used to study the $\eta-\eta^{\prime}$ mixing angle, which is important to test $\mathrm{SU}(3)$ flavor symmetries in QCD.

We report the observation (evidence) of the $h_{c}$ radiative decay $h_{c} \rightarrow \gamma \eta^{\prime}(\eta)$, where $h_{c}$ is produced in the decay $\psi^{\prime} \rightarrow \pi^{0} h_{c}$. The $h_{c} \rightarrow \gamma \eta^{\prime}$ is reconstructed by using $\eta^{\prime} \rightarrow \pi^{+} \pi^{-} \eta$ with $\eta \rightarrow \gamma \gamma$ and $\eta^{\prime} \rightarrow \gamma \pi^{+} \pi^{-}$. The $h_{c} \rightarrow \gamma \eta$ is reconstructed from decays $\eta \rightarrow \gamma \gamma$ and $\eta \rightarrow \pi^{+} \pi^{-} \pi^{0}$ with $\pi^{0} \rightarrow \gamma \gamma$. The analyses [6] are based on a data sample of $4.48 \times 10^{8} \psi^{\prime}$ events collected with the BESIII detector.

Figure 2 shows the distributions of the invariant masses $M\left(\gamma \eta^{\prime}\right)$ and $M(\gamma \eta)$ for the selected events. Signals for the $h_{c}$ meson are observed. In order to extract the signal yield a simultaneous maximum likelihood fit is performed on $\eta^{\prime} \rightarrow \pi^{+} \pi^{-} \eta$ and $\eta^{\prime} \rightarrow \gamma \pi^{+} \pi^{-}$events for the $h_{c} \rightarrow \gamma \eta^{\prime}$ decay, and on $\eta \rightarrow \gamma \gamma$ and $\eta \rightarrow \pi^{+} \pi^{-} \pi^{0}$ events for the $h_{c} \rightarrow \gamma \eta$ decay, respectively. The signal shape is modeled using signal MC events and the background is described with the ARGUS function. In Table 2 the results are summarized.
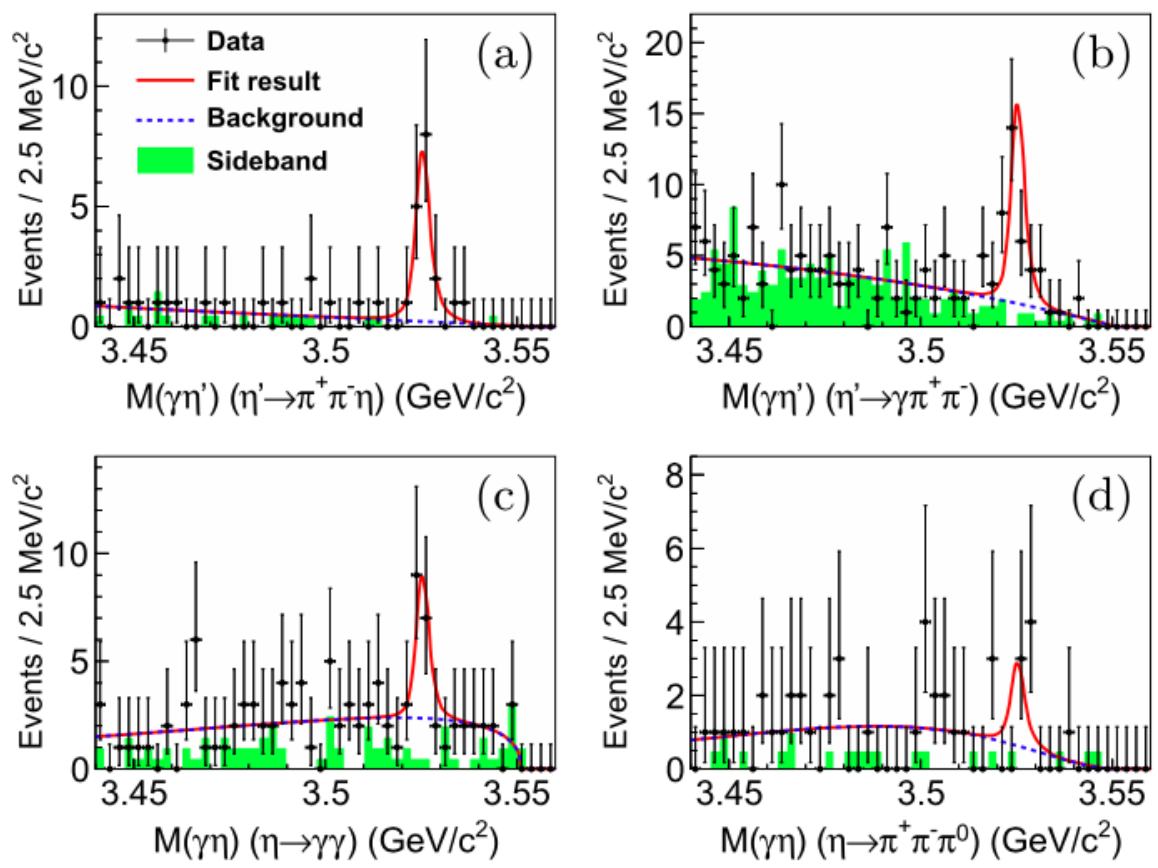

Figure 2: Results of the simultaneous fits to the invariant mass distributions. The red solid curves are the fit results, the blue dashed curves are the background distributions, and the green histograms are events from $\eta^{\prime}(\eta)$ sidebands. 
Table 2: Results on $h_{c} \rightarrow \gamma \eta^{\prime}(\eta)$.

\begin{tabular}{lrrrr}
\hline Mode & $N_{h_{c} \rightarrow \gamma \eta^{\prime}(\eta)}$ & $\mathscr{B}\left[h_{c} \rightarrow \gamma \eta^{\prime}(\eta)\right]$ & Significance & $\mathscr{B}\left(h_{c} \rightarrow \gamma \eta\right) / \mathscr{B}\left(h_{c} \rightarrow \gamma \eta^{\prime}\right)(\%)$ \\
\hline$h_{c} \rightarrow \gamma \eta^{\prime} 44.3 \pm 7.8$ & $(1.52 \pm 0.27 \pm 0.29) \times 10^{-3}$ & $8.4 \sigma$ & $30.7 \pm 11.3 \pm 8.7$ \\
$h_{c} \rightarrow \gamma \eta$ & $18.1 \pm 5.8$ & $(4.7 \pm 1.5 \pm 1.4) \times 10^{-4}$ & $4.0 \sigma$ & \\
\hline
\end{tabular}

\section{Study of $\chi_{c J}$ decaying into $\phi K^{*}(892) \bar{K}$}

Unlike the $J / \psi$ and $\psi(3686)$, the $P$-wave charmonia states $\chi_{c J}$ are not directly produced in $e^{+} e^{-}$collisions and, thus, are less well understood to date. Obtaining more experimental data on exclusive decays of these $\chi_{c J}$ states is important for a better understanding of their nature and decay mechanisms, as well as for testing QCD-based calculations. Since the $\chi_{C J}$ states are produced copiously in the electric dipole (E1) radiative transition of $\psi(3686)$, with branching fractions around $9 \%$, the large $\psi(3686)$ data sample taken at BESIII provides a unique opportunity for detailed studies of $\chi_{c J}$ exclusive decays.

In this analysis [7] we report the first measurement of the decay $\chi_{c J} \rightarrow \phi K_{S}^{0} K^{ \pm} \pi^{\mp}$ and $\chi_{c J} \rightarrow$ $\phi K^{+} K^{-} \pi^{0}$ in the E1 radiative transition $\psi(3686) \rightarrow \gamma \chi_{c J}$. The $\phi$ is reconstructed via $K^{+} K^{-}$. The analysis is based on a data sample of $1.06 \times 10^{8} \psi(3686)$ events collected with the BESIII detector.

The invariant mass distributions of $K^{+} K^{-} K_{S}^{0} K^{ \pm} \pi^{\mp}$ and $K^{+} K^{-} K^{+} K^{-} \pi^{0}$ are shown in Figure 3. Significant $\chi_{c J}$ signals are observed with low background. The dominant processes are the $\chi_{c J} \rightarrow \phi K^{*}(892) \bar{K}$ three-body decays. The branching fractions for this reaction via neutral and charged $K^{*}(892)$ are measured for the first time and are summarized in Table 3.

By examining the invariant mass spectrum of $K \bar{K} \pi$, a significant excess of events above the phase space expectation is observed near the $K^{*}(892) \bar{K}$ mass threshold in the decays of $\chi_{c 1,2}$ with a significance greater than $10 \sigma$. The observed structure has negative $\mathrm{C}$ parity, and is expected to be the $h_{1}(1380)$ state, considering its mass, width and decay through $K^{*}(892) \bar{K}$. In the Figure 4 is shown the simultaneous fit performed to the invariant mass distributions of $K \bar{K} \pi$ for the candidate events in the $\chi_{c 1,2}$ signal regions. The mass and the width of the $h_{1}(1380)$ are determined to be $1412 \pm 4 \pm 8 \mathrm{MeV} / \mathrm{c}^{2}$ and $84 \pm 12 \pm 40 \mathrm{MeV}$ respectively. This is the first direct observation of the $h_{1}(1380)$ in its decay to $K^{*}(892) \bar{K}$. Evidence is also found for the decays $\chi_{c J} \rightarrow \phi \phi(1680)$ and $\chi_{c J} \rightarrow \phi \phi(1850)$ but with significances less than $5 \sigma$.

More data and advanced analysis techniques may shed light on the properties of the structures observed in the $K \bar{K} \pi$ invariant mass spectrum

Table 3: Branching fractions measured in $\phi K \bar{K} \pi$ final states.

\begin{tabular}{rrrr}
\hline & Decay Modes & $\phi K_{S} K^{ \pm} \pi^{\mp}\left(\times 10^{-3}\right)$ & $\phi K^{+} K^{-} \pi^{0}\left(\times 10^{-3}\right)$ \\
\hline$\chi_{c 0}$ & $\phi K^{*}(892)^{ \pm} K^{\mp}$ & $1.65 \pm 0.21 \pm 0.22$ & $1.90 \pm 0.14 \pm 0.32$ \\
& $\phi K^{*}(892)^{0} \bar{K}^{0}+$ c.c. & $2.03 \pm 0.21 \pm 0.28$ & $\ldots$ \\
$\chi_{c 1}$ & $\phi K^{*}(892)^{ \pm} K^{\mp}$ & $1.76 \pm 0.21 \pm 0.26$ & $1.62 \pm 0.12 \pm 0.28$ \\
& $\phi K^{*}(892)^{0} \bar{K}^{0}+$ c.c. & $1.51 \pm 0.19 \pm 0.22$ & $\ldots$ \\
$\chi_{c 2}$ & $\phi K^{*}(892)^{ \pm} K^{\mp}$ & $2.56 \pm 0.23 \pm 0.35$ & $2.74 \pm 0.16 \pm 0.44$ \\
& $\phi K^{*}(892)^{0} \bar{K}^{0}+$ c.c. & $2.27 \pm 0.22 \pm 0.32$ & $\ldots$ \\
\hline
\end{tabular}



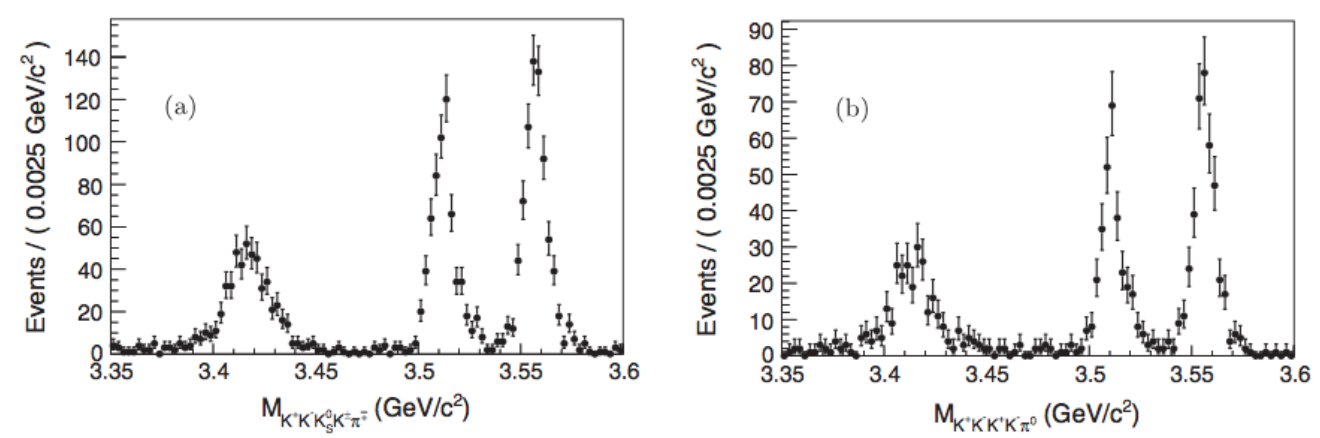

Figure 3: Invariant mass distribution of (a) $K^{+} K^{-} K_{S}^{0} K^{ \pm} \pi^{\mp}$ and (b) $K^{+} K^{-} K^{+} K^{-} \pi^{0}$.

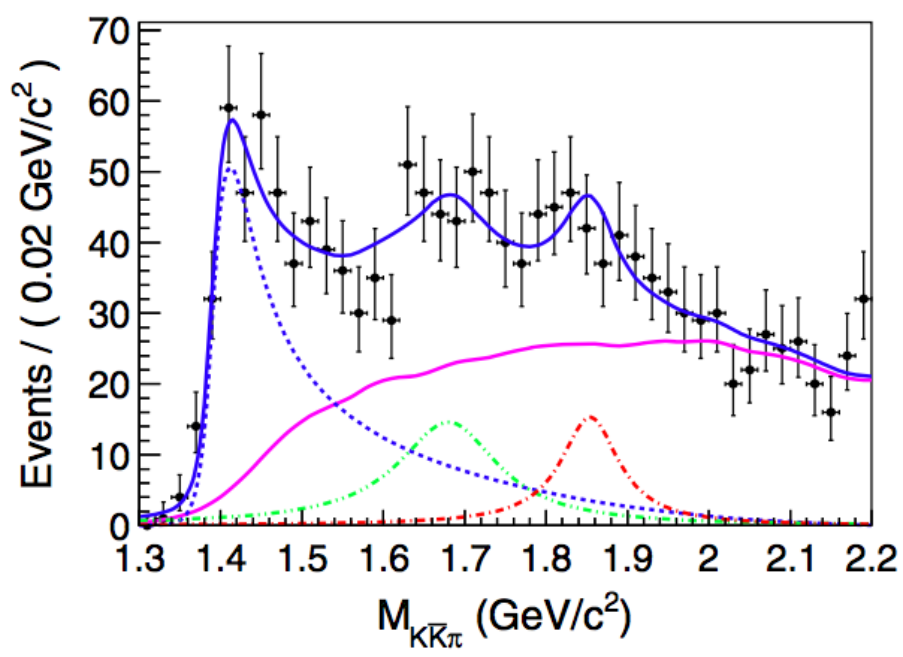

Figure 4: The sum of $K_{S} K^{ \pm} \pi^{\mp}$ and $K^{ \pm} K^{\mp} \pi^{0}$ mass spectra in the $\chi_{c 1}$ and $\chi_{c 2}$ mass regions. The markers with error bars represent the data; the dash curve the $h_{1}(1380)$ signal; the dash-dot-dot curve the $\phi(1680)$ signal; and the dash-dot curve the $\phi(1850)$ signal.

\section{References}

[1] B. Auber et al., Phys. Rev. D 69 (2014) 011103R.

[2] G. S. Adamas et al., Phys. Rev. D 73 (2006) 051103R.

[3] V. V. Anashin et al., Phys. Lett. B 695 (2010) 134-140.

[4] M. Ablikim et al., Phys. Lett. B, 761 (2016) 98-103.

[5] M. Ablikim et al., Phys. Rev. D 88 (2013) 032007.

[6] M. Ablikim et al., Phys. Rev. Lett. 116 (2016) 251802.

[7] M. Ablikim et al., Phys. Rev. D 91, (2015) 112008. 